\title{
El Patrimonio cultural al servicio de prácticas de Educación Permanente en entornos barriales. El caso de Playa Ancha - Valparaíso
}

\author{
Marco Muñoz-del Campo \\ mmunozc@upla.cl \\ Doctor en Educación Permanente \\ Universidad de Playa Ancha-Valparaíso, Chile
}

Recepción:06 de abril del 2021

Aprobación:28 de mayo del 2021

Publicación: 06 de junio del 2021

\section{Resumen}

Desde la inscripción de la Zona Histórica de la ciudad portuaria de Valparaíso - Chile en la Lista del Patrimonio Mundial, como resultado del proceso de seguimiento que se lleva a cabo en todos los sitos inscritos, el Consejo Internacional de Monumentos y Sitios viene insistiendo en la creciente necesidad de fortalecer la identidad cívica, en lo relativo a la interpretación del Valor Universal Excepcional del bien inscrito y la participación en ello, de los principales actores de la ciudad. En tal sentido, urge atender el arraigo de la comunidad a su territorio, como depositario de diversos valores patrimoniales que no han sido debidamente estudiados, informados y transferidos. Ante esta situación problemática, bajo el paradigma de la investigación-acción participativa, mediante una metodología prospectiva y la sistematización como estrategia de aprendizaje a partir de la práctica; se presenta en este artículo, una experiencia de educación permanente, desarrollada en el espacio público del cerro Playa Ancha, tendiente a la construcción de conocimiento compartido por un grupo de habitantes; quienes, como resultado de este proceso, logran empoderarse de la responsabilidad de significar, fortalecer y transferir los valores patrimoniales de su territorio.

Palabras claves. Patrimonio cultural, educación permanente, valores patrimoniales, participación social, prospectiva, sistematización. 


\title{
Cultural heritage at the service of Permanent Education practices in neighborhood environments. The case of Playa Ancha -Valparaíso
}

\begin{abstract}
Since the inscription of the Historic Zone of the port city of Valparaíso - Chile on the World Heritage List, as a result of the monitoring process carried out in all the registered sites, the International Council of Monuments and Sites has been insisting on the growing need to strengthen civic identity, regarding the interpretation of the Outstanding Universal Value of the inscribed property and the participation in it, of the main actors of the city. In this sense, it is urgent to attend to the roots of the community in its territory, as a depository of various heritage values that have not been duly studied, informed and transferred.

Faced with this problematic situation, under the paradigm of participatory action research, through a prospective methodology and systematization as a learning strategy from practice; this article presents an experience of permanent education, developed in the public space of Playa Ancha hill, aimed at the construction of shared knowledge by a group of habitants; who, as a result of this process, manage to empower themselves with the responsibility to signify, strengthen and transfer the heritage values of their territory.
\end{abstract}

Keywords. Cultural heritage, permanent education, heritage values, social participation, prospective, systematization.

\section{Introducción}

Ante la necesaria acción transformadora de orden social, que requiere la ciudad de Valparaíso, con el propósito de revertir la forma en que se aborda la gestión patrimonial anclada en la institucionalidad representada en las autoridades regionales y comunales, donde la participación no logra vencer la distancia entre quienes gobiernan, administran e intervienen el patrimonio cultural y aquellos que forman parte de su dinámica social, como habitantes y protagonistas del acontecer que dinamiza la ciudad; esta investigación se desarrolló a partir de la aplicación de técnicas dialógicas en un grupo de vecinos del barrio de Playa Ancha Bajo y dialécticas con el espacio público, para la necesaria construcción de conocimiento en torno a la 
significación y re-significación de valores patrimoniales que subyacen en las expresiones materiales e inmateriales que conforman el lugar que habitan.

Como antecedente del proceso de intervención que describe el presente artículo y como resultado de un estudio realizado el año 2014, que llevó a cabo un análisis, precisamente, del uso con fines educativos del Patrimonio Cultural de Valparaíso, a partir del concepto de ciudad educadora, se concluye que "es urgente plantear acciones sobre la valoración del patrimonio de la ciudad, porque no existe un trabajo dirigido a la formación de la ciudadanía en el valor y respeto por la historia local a partir del conocimiento del patrimonio presente en la ciudad”. (Dámaris, 2014)

Tal como plantea la Organización de las Naciones Unidas para la Educación, la Ciencia y la Cultura en el texto de la Convención para la Protección del Patrimonio Mundial (UNESCO, Convención para la proteccción del Patrimonio Mundial, 1972) y que a su vez, vuelve a consignar la Carta de Ciudades Educadoras (Ciudades Educadoras, 1990); lo relevante en los procesos de toma de conciencia, por parte de la ciudadanía, respecto de la condición patrimonial de cada sitio y la responsabilidad que asume cada Estado en una adecuada gestión del patrimonio cultural, es propender a la generación y difusión de conocimiento compartido, a la participación responsable de los ciudadanos en la gestión del bien y a la implementación de acciones educativas tendientes a preservarlo (Ballart \& i Tresseras, 2001).

En este sentido, el Consejo Internacional de Monumentos y Sitios, ha observado permanentemente, al Estado chileno, la necesidad de fortalecer la identidad cívica y para ello se hace necesario diseñar e instalar procesos de concienciación, respecto del Valor Universal Excepcional del bien inscrito, donde no puede quedar ausente la ciudadanía. (UNESCO, Casco Histórico de la Ciudad Portuaria de Valparaíso, 2014-2019)

En este contexto, urge un compromiso del Estado con los individuos y las comunidades que conforman el territorio, en cuanto a buscar -a través de la educación- maneras de descubrir los valores del patrimonio cultural de la Nación, entendidos como un contenido de aprendizaje básico que debe provocar una reflexión acerca de ellos e internalizarlos conscientemente, haciendo de la educación un proceso abierto y participativo tendiente a la protección y fortalecimiento de los bienes culturales.

$\mathrm{Al}$ respecto, algunos autores hablan del valor educativo del patrimonio, que para efectos de la categorización de valor que hacen Ballart \& i Tesseras (2001, pág. 20), podría estar incluido en 
los valores de uso inmaterial; pero, ante el peligro que pueden significar para la conservación del patrimonio cultural aquellas interpretaciones parciales o equívocas de nuestra herencia, aparece como algo relevante, evaluar de manera especial los bienes en función de su "capacidad de suscitar una toma de conciencia sobre la cultura y la historia como medio para integrar los bienes culturales al presente” (Losada Aranguren, 1997,124).

María Teresa Devia (2003) señala que la educación en torno al patrimonio, a partir de su marcado valor social, debe resolver dos aspectos que le dan sentido a la vida de las personas en comunidad y en armonía con el entorno. Por un lado el de "fortalecer el sentimiento de ser acogido como miembro de un grupo social (pertenencia) y por otro el de identificarse con sus costumbre y tradiciones (identidad).” (pág. 53).

Desde un punto de vista más político Mauricio Suazo (2003) señala que la educación, entendida como un bien o servicio al cual toda persona tiene derecho, debe responder a dos exigencias fundamentales, "la de permitir a las personas desarrollar sus capacidades individuales y la de satisfacer las necesidades y demandas de una sociedad, en términos de desarrollo y progreso” (pág. 18). En tal sentido, se debe considerar la educación como una inversión, cuyos beneficios económicos, culturales y sociales, deben verse reflejados en la sociedad a la que se pretende educar.

Es así que este estudio se sustenta en aquellos enfoques cuya concepción de la educación se construye a partir de procesos que involucran el sistema social en forma integral y que en tal sentido, podrían contribuir a la concienciación y a la preservación de los valores culturales de la ciudad de Valparaíso.

Por ejemplo, la Pedagogía Social, definida como "la didáctica de las relaciones sociales, que supone en ella un rol socializador, dirigido a la adquisición de competencias, la participación social, la prevención de problemáticas y conflictos y a una mayor permeabilidad de los ámbitos institucionales educativos frente a sus entornos sociales de diverso orden" (Petrus, 1997) y fundada en un conjunto de experiencias y acciones educativas vinculadas socialmente que deben ser puestas en práctica; podría contribuir a concretar la intención manifestado desde 2005 por el Centro de Estudios Sociales de la ciudad de Valparaíso, en orden a "Instalar una estrategia pedagógica formativa que promueva el conocimiento y la valoración que tienen niños y jóvenes respecto de los símbolos del patrimonio cultural y social, elementos que permitirían comprender la realidad social y cultural de su entorno y participar en ella comprendiendo claramente qué se 
va a entender por patrimonio cultural y social, cuál es la realidad social y cultural del entorno y cuál será el contenido de aquello enunciado como lo identitario" (Centro de Estudios Sociales CIDPA, 2005, pág. 2)

Entre estas nuevas tendencias, se encuentra también la invitación que hace el Centro Internacional de Prospectiva y Altos Estudios del Estado de Puebla - México - CIPAE, en cuanto a percibir la educación como un movimiento personal y social permanente; como una evolución de la humanidad. Evolución que compromete justamente las relaciones individuales y grupales, consigo mismo, con el resto de la sociedad y con el entorno. Se trata de la Educación Permanente, entendida como un proceso de transformación consciente de las relaciones sociales, transformación deseada e intencionada que humaniza y que por ello tiene la potencialidad de hacer realizable el desarrollo como un crecimiento armónico individual y social.(CIPAE, 2012).

La Educación Permanente es un concepto derivado de una concepción educativa centrada en la "construcción continua de la persona humana, de su saber y de sus aptitudes, pero también de su capacidad de juicio y de decisión”, una “experiencia única e intransferible y en la cual la persona se apropia de conocimientos, construye saberes, adquiere nuevas competencias, mejora sus desempeños sociales, enriquece sus relaciones con los demás y con su entorno y consigo misma”. (CIPAE, 2012) Una práctica reiterada, sistemática e intencionalmente desarrollada por los sujetos y que constituye un proceso orientado en cuatro grandes direcciones: aprender a aprender, aprender a hacer, aprender a ser y aprender a convivir. "(...) un proceso continuo que permite apropiarse críticamente de los saberes, competencias, actitudes y destrezas necesarias para comprender la realidad, penetrarla, valorar su universo simbólico y darle sentido a los eventos y circunstancias de su cotidianidad. En tal sentido, sugiere desbordar los límites de la educación formal y copar todos los espacios y ambientes de la sociedad”. (Mondaca, 1997, pág. 10)

Sin embargo, la aplicación de estos enfoques es dependiente de la política educativa de orden social que, a decir de Luis Benavides, director y creador de CIPAE, “oriente las decisiones y las acciones educativas hacia modelos no excluyentes, en beneficio permanente de toda la sociedad, y porque pretende incluir en los procesos educativos todas las instancias y organismos sociales"(CIPAE, 2012).

En este contexto, nuestra sociedad está llamada a buscar y crear nuevos espacios educativos y nuevos diseños metodológicos, ya que la educación a lo largo de la vida y en todos los espacios 
de interacción social, conduce directamente a la noción de sociedad educativa, es decir, a una sociedad dispuesta a aprender y a la que se debe ofrecer múltiples posibilidades que, no necesaria y exclusivamente, deben desarrollarse en espacios asociados al concepto de aula, con muros reales y simbólicos que aíslan a los individuos de los distractores del entorno. Entendiendo que hoy, la educación exige que los espacios educativos concurran a una construcción solidaria del saber y que promuevan el intercambio al interior y exterior. Que sean espacios donde se pueda producir un conocimiento en comunidad y donde se pueda analizar y resolver problemas comunes.

Entendida la educación como un servicio público, se debe propender a que los espacios educativos sean públicos y que los espacios públicos puedan ser espacios educativos, donde las personas puedan experimentar no sólo aprendizajes curriculares, sino también aprendizajes que la educación moderna exige. Entre ellos, la formación cultural centrada en el fortalecimiento de valores patrimoniales arraigados a un determinado grupo humano y que bajo una visión psicagógica $^{1}$ del espacio, no pueden ser completamente comprendidos si no se estudian en el contexto en el que se manifiestan (Benavides L., 2002, págs. 1-2).

Sustentada en esta concepción espacial de la experiencia de educarse, la tesis central que plantea este estudio, recoge lo expresado por Luis Benavides en el Taller Internacional sobre Infraestructura Educativa, desarrollado en la ciudad de Guadalajara México en 2002, en tanto adopta el siguiente supuesto implícito: “(...) los espacios creados por el ser humano, por sí mismos educan, ya que configuran el tipo de ser humano que vive, se desarrolla y se 'acomoda' en él." (Benavides L. , 2002, págs. 1-2)

\section{Problema de Investigación}

Relevando la responsabilidad que le cabe a las universidades en la educación de valores patrimoniales, pero a partir de un enfoque inclusivo e interdependiente, en permanente diálogo con la comunidad y considerando la oportunidad de inscribir esta investigación en un proceso de vinculación con el medio iniciado por la Universidad de Playa Ancha en 2014 -basado en la asociación de los distintos actores del territorio, como elementos determinantes de su propio desarrollo, donde ha sido comprobada la factibilidad de desarrollar experiencias de participación social y donde se ha detectado un alto interés de la comunidad en torno a construir conocimiento

\footnotetext{
1 "Estudio de la fuerza atractora o repulsora que tienen los espacios para guiar los sentimientos, los afectos o desafectos de las personas. En esta rama del saber, los espacios tienen una funcionan que invita a la acción o la pasión, como es fácil comprobarlo en la multitud de espacios arquitectónicos intencionados que hoy día se construyen".
} 
compartido respecto de los valores patrimoniales del barrio- se decidió iniciar un proceso de intervención, bajo el enfoque de la Investigación-Acción Participativa, a partir de la siguiente interrogante:

¿Cómo desarrollar procesos de aprendizaje orientados a la formación de valores patrimoniales, a partir de una experiencia de educación permanente en el sector de Playa Ancha Bajo, territorio que posee características sociales, culturales y naturales de un reconocido valor patrimonial?

Bajo el supuesto que reconoce la existencia de espacios educativos en el espacio público, a partir de una concepción socializadora de la educación que hace permeables los muros reales y simbólicos que aíslan a las personas del entorno social y cultural donde se manifiestan las expresiones valoradas por un determinado grupo humano, se pretende lograr procesos de aprendizaje que surjan de la experiencia de habitar los espacios públicos que construyen el entorno, mediante la aplicación de prácticas educativas sustentadas en los pilares de la educación permanente; como son, la participación social, la prospectiva y la sistematización de la práctica educativa.

De esta forma se configura el planteamiento hipotético bajo el supuesto que mediante procesos de aprendizaje compartido en el espacio público; es posible promover en los ciudadanos actitudes y cualidades que les permitan comprender y tomar conciencia de la significación del patrimonio cultural presente en su territorio y de esta manera, experimentar un proceso formativo que contribuye al fortalecimiento de la identidad cívica, mediante el conocimiento, uso y goce de los valores patrimoniales del lugar que habitan.

\section{Objetivos}

Re-conocer espacios públicos educativos en el barrio de Playa Ancha Bajo e identificar los valores patrimoniales que subyacen en las expresiones que construyen el entorno social y cultural.

Contribuir al diseño de un modelo de educación permanente para la formación de valores patrimoniales, a partir del registro, puesta en valor y evaluación de una experiencia de participación social educativa.

\section{Metodología}

El trabajo investigativo presentado en este artículo, consistió en aplicar métodos conducentes a incrementar y fortalecer en los habitantes el sentido de apropiación, la relación de 
pertenencia, la concienciación, el respeto a la tradición, el valor de la historia, la responsabilidad frente a la necesidad de educarse y, por consiguiente, hacerse cargo del desarrollo individual y colectivo de la comunidad que representan y del lugar donde viven. Lugar que a diario habitan, en tanto construyen y cuidan (Heiddeger, 2004) con el propósito de heredar un mejor futuro para sus descendientes.

Tomando en cuenta la necesidad de poner en práctica un método de generación de conocimiento compartido respecto de la realidad, durante y mediante un proceso de transformación intencionada, pero integrando como parte esencial al propio sujeto de la transformación, entendido como gestor y autor del conocimiento que se pretende lograr; se decidió orientar la ejecución de la investigación a partir de un enfoque que reúne acción transformadora y participación efectiva, como es el de la Investigación-Acción Participativa. Enfoque cuya trascendencia radica en la práctica de lo consciente, de donde deriva el "conocimiento necesario para la transformación” de la sociedad, acompañado además, de la "construcción de saberes que apuntan a un conocimiento científico" (Schmelkes, 2011, pág. 78). ${ }^{2}$

Lo que plantea su promotor Fals Borda, según Silvia Schmelkes (2011) es que, bajo este paradigma alternativo, es posible lograr la construcción de conocimientos en forma compartida, respondiendo a intereses de transformación social real, a través de una práctica educativa que potencia el poder de los habitantes de un territorio determinado, en tanto lograr la capacidad de reconocerse e identificarse con el patrimonio cultural del lugar que habitan. De esta manera, asumir un liderazgo en la preservación de sus valores, heredando a las generaciones venideras, no tan solo su expresión física y simbólica, sino además, legando su propia experiencia, la que también se vuelve heredable, en tanto forma de educarse con y para el patrimonio; instalando en la comunidad, el hábito de aprender a conocerlo [conocer], construirlo [hacer], compartirlo [convivir] y valorarlo [ser]. (Benavides L. , 2002, págs. 1-2)

\section{La prospectiva y la transformación de la realidad}

La prospectiva, uno de los pilares que sostiene la filosofía de la educación permanente, persigue instalar en los individuos la responsabilidad de educarse como un proceso inherente a su existencia, asumiendo una actitud capaz de transformar el sistema de relaciones que lo vinculan con su entorno humano, físico y simbólico, en un proceso de toma conciencia respecto del hoy,

\footnotetext{
${ }^{2}$ Cita del autor: Ver FALS, Borda (1985) Orlando: “Comentario" o Marcela Gajardo. En: Gajardo M. (Comp) “Teoría y Práctica de la Educación Popular”. Pátzcuaro. Mich. CREFAL-OEA-IDRC.
} 
vivenciando el pasado desde una percepción actual que les permita prefigurar escenarios deseables $\mathrm{y}$, en virtud de ello, definir y concretar acciones para modificar el futuro en su estado presente.

Este proceso permite aprendizajes individuales y colectivos que en forma permanente -a partir de una reflexión y toma de conciencia- posibilitan identificar fortalezas y debilidades y, desde ahí, definir los recursos con los que se cuenta para modificar la realidad, superando los obstáculos que plantean las diversas situaciones internas y externas en el devenir de una comunidad. Así, en un determinado contexto de reflexión, en busca de una mejora o solución, es posible comprender los retos que exige el proceso de acercamiento del futuro y determinar las herramientas con las que se cuenta para actuar sobre él.

Los individuos quedan entonces, empoderados de una comprensión de la realidad, de los impactos que ésta genera en su devenir y de lo que son capaces de hacer con ella; adquieren la capacidad de acercar el futuro y de manera intencional e informada, prefigurar escenarios que permiten en una primera instancia, situarse en lo que podría suceder de ser asumida una actitud prospectiva para producir un cambio. Se produce una construcción de conocimiento respecto del futuro, sustentado en la interacción dialógica y reflexiva, dando lugar a un proceso de comprensión que los sitúa como actores en escenarios que todavía no suceden, respecto de los cuales es posible tomar decisiones de momento que se es capaz de "presencializar las distintas posibilidades de futuro con relación a la acción presente y a las diferentes alternativas de modificación de la misma”. (Benavides L. , Metodología Prospectiva, 2004 a)

El desarrollo de la metodología prospectiva propone la construcción o prefiguración de tres escenarios, el escenario probable, el escenario deseable y el escenario posible, que respectivamente refieren, "un futuro que tiene mayores posibilidades de ocurrir", si lo que está sucediendo permanece inalterado; aquel que se configura a partir del "ideal que se desea", omitiendo los obstáculos que surgen de la confrontación con la realidad y, finalmente, aquel que "conjuga las premisas realizables de lo deseable con lo lograble, para conformar lo posible -no sin esfuerzodentro de lo deseable”. (Benavides L. , Metodología Prospectiva, 2004 a)

De esta forma, una vez anticipado el futuro posible, con la debida concienciación de la realidad individual y colectiva, en tanto conocedores de los recursos con los que se cuenta, lo que se es y lo que se quiere ser y llegar a ser a partir de hoy, los individuos de un determinado grupo humano, frente a una determina realidad, quedan en condiciones de tomar decisiones para actuar desde el momento presente, concretando sistemáticamente acciones transformadoras que se van 
desarrollando, evaluando y modificando en el tiempo, como parte de un proyecto de largo plazo, pero que comienza hoy.

Frente a la acción transformadora, la prospectiva opera de tal forma que un conjunto de individuos logra comprender y transformar la realidad, a favor del desarrollo social, con el fin de anticiparse y actuar sobre su futuro, considerando para ello dos aspectos fundamentales: en primer lugar, la construcción de prefiguraciones, en base a objetivos comunes -donde aparece como necesidad fundamental considerar espacios de diálogo que permitan la conformación de comunidades empoderadas y capaces de transitar más allá de soluciones que se agotan en sí mismas- y, en segundo lugar, la de desarrollar y fortalecer día a día una actitud, una forma de ser, de hacer, de convivir y de actuar sobre esos escenarios futuros. Anticipándose al devenir de la sociedad, actuando oportunamente para transformar la realidad y garantizar un mejor futuro para las nuevas generaciones.

Es así como surge la posibilidad de provocar una sinergia entre la educación permanente y la preservación y fortalecimiento del patrimonio cultural, sustentando la metodología utilizada, en prácticas educativas centradas en indagar y aproximarse a los valores que subyacen en aquella construcción social heredada y que nacen de la necesaria relación sujeto-realidad que los individuos logran establecer, descubrir y comprender. Se establece que el patrimonio no es algo físico que se encuentra allí esperando ser atendido, sino que se encuentra, se interpreta, se descubre y se comprende desde la necesaria relación consciente de las personas con aquella manifestación física o simbólica.

Así entonces, sobre la base de técnicas dialógicas de participación social y de una puesta en valor de la experiencia, mediante la sistematización como estrategia de aprendizaje a partir de la práctica para la generación de conocimiento compartido (Ruiz Botero, 2001), el enfoque prospectivo permitió proporcionar al estudio un sustento metodológico que hizo posible el proceso de investigación-acción participativa propuesto.

\section{Dialéctica y dialógica para el aprendizaje compartido en el espacio público}

Para producir una orientación adecuada a la práctica que se deseaba realizar, utilizando técnicas que permitieran aportar fundamentalmente al para qué de la experiencia, sobre la base de la actitud prospectiva que se deseaba lograr en los actores, motivando un nivel de participación que los hiciera posesionarse y compartir sus vivencias, su experiencia pasada puesta en valor a través de la experiencia presente; hubo que revisar distintas técnicas que proporciona el paradigma 
cualitativo, entre ellas, el diálogo discusión en distintos formatos (panel, mesa redonda, discusión dirigida, grupos de discusión abierta, revisión documental comentada o grupos focales). Asimismo, se revisaron algunas formas de aplicar la técnica de ejercicio vivencial (seminario, estudio de casos, teatro o disco foro, juego de roles, experiencia estructurada, aprendizaje cooperativo, excursión, juego, simulación, aprendizaje a partir de proyectos, ensayo, entrevistas o visitas guiadas).

De todas ellas se optó por los Grupos de Discusión Abierta y la técnica de Excursión (Valles, 2003). A pesar que esta última es de carácter instruccional y en virtud de ello, no respondería a los objetivos del estudio, se utilizó con una orientación distinta, a partir de la experiencia del investigador, en cuanto a su desempeño como docente en asignaturas de Taller de Diseño Arquitectónico, donde se utiliza técnicas de aprendizaje basadas en el estudio de la realidad, a partir de una técnica muy parecida a la excursión y que consiste en visitarla, observarla y registrarla para reflexionar respecto de ella antes, durante y luego de haberla visitado, con el objetivo de interpretarla y comprenderla desde la memoria, la observación y la reflexión, para luego, actuar sobre ella y transformarla.

\section{Etapas del proceso}

La experiencia se desarrolló con la participación de un conjunto de 38 vecinos del barrio de Playa Ancha Bajo, en una actividad que se denominó "Talleres de Participación Social: Aprendiendo en el espacio público a través de la experiencia” y que contempló tres etapas y las siguientes actividades:

Tabla 1 Etapas y actividades desarrolladas.

\begin{tabular}{|l|l|l|}
\hline \multicolumn{2}{|c|}{ Etapas } & \multicolumn{2}{c|}{ Actividades } \\
\hline $\begin{array}{l}\text { Fiagnóstico } \\
\text { participativo del } \\
\text { estado de } \\
\begin{array}{l}\text { conciencia del } \\
\text { conjunto social, } \\
\text { respecto de los } \\
\text { valores } \\
\text { patrimoniales del } \\
\text { lugar que habitan }\end{array}\end{array}$ & A1 & $\begin{array}{l}\text { Registro descriptivo por grupos de discusión, de los lugares } \\
\text { donde subyace el valor patrimonial del barrio de Playa Ancha } \\
\text { Bajo, a partir de un proceso de reconstrucción histórica con } \\
\text { foco en la experiencia de habitar }\end{array}$ \\
\hline $\begin{array}{l}\text { A3 } \\
\text { Construcción de una Cartografía Participativa del Patrimonio } \\
\text { Valorado integrando el trabajo de cada grupo }\end{array}$ \\
\hline $\begin{array}{l}\text { Descripción y categorización del valor patrimonial del barrio, } \\
\text { desde el sistema de relaciones que vinculan a los habitantes } \\
\text { entre sí y con los lugares identificados }\end{array}$ \\
\hline
\end{tabular}




\begin{tabular}{|c|c|c|c|}
\hline & & A4 & $\begin{array}{l}\text { Construcción colectiva de una Cartografía Prospectiva del } \\
\text { Patrimonio Sistematizado integrando el trabajo de cada grupo }\end{array}$ \\
\hline \multirow{2}{*}{ F2 } & \multirow{2}{*}{$\begin{array}{l}\text { Excursión por el } \\
\text { barrio visitando los } \\
\text { lugares heredables } \\
\text { reconocidos }\end{array}$} & A5 & $\begin{array}{l}\text { Diseño de Itinerarios temáticos por ámbitos de valor. Estético } \\
\text { Formal, Asociativo Simbólico y de Uso Social (Ballart \& i } \\
\text { Tresseras, 2001) }\end{array}$ \\
\hline & & A6 & $\begin{array}{l}\text { Re-significación de aquellos valores encontrados a través del } \\
\text { proceso de reconstrucción histórica, incorporando la vivencia } \\
\text { individual y colectiva en el presente }\end{array}$ \\
\hline \multirow{4}{*}{ F3 } & \multirow{4}{*}{$\begin{array}{l}\text { Propuesta de } \\
\text { acciones } \\
\text { transformadoras, } \\
\text { tendientes a actuar } \\
\text { sobre el futuro del } \\
\text { patrimonio del } \\
\text { barrio }\end{array}$} & A7 & $\begin{array}{l}\text { Descripción e Identificación, individual, de los lugares } \\
\text { patrimoniales, su significación y su puesta en valor prospectiva }\end{array}$ \\
\hline & & A8 & $\begin{array}{l}\text { Prefiguración descriptiva de escenarios futuros, reflejando en } \\
\text { una ficha de registro individual, lo probable (lo que podría } \\
\text { suceder si no se valora), lo deseable (lo que se desearía } \\
\text { suceda) y lo posible (lo que se podría hacer para favorecer lo } \\
\text { deseable y evitar lo posible) }\end{array}$ \\
\hline & & A9 & $\begin{array}{l}\text { Registro individual de acciones prospectivas orientadas a } \\
\text { proteger e incrementar el patrimonio cultural del barrio }\end{array}$ \\
\hline & & A10 & Propuesta de proyectos prospectivos por grupos de discusión \\
\hline
\end{tabular}

El proceso comenzó en un espacio facilitado por una junta vecinal, donde los habitantes, organizados en tres grupos de discusión, desarrollaron un ejercicio de reconstrucción histórica, acudiendo a la memoria -centrando el ejercicio en la experiencia de reconocerse como parte y habitante de un territorio- a objeto de recordar e identificar aquellas manifestaciones físicas y simbólicas situadas en el espacio público, donde han construido y desarrollado su vida en comunidad. 
Figura 1. Grupos de Discusión.

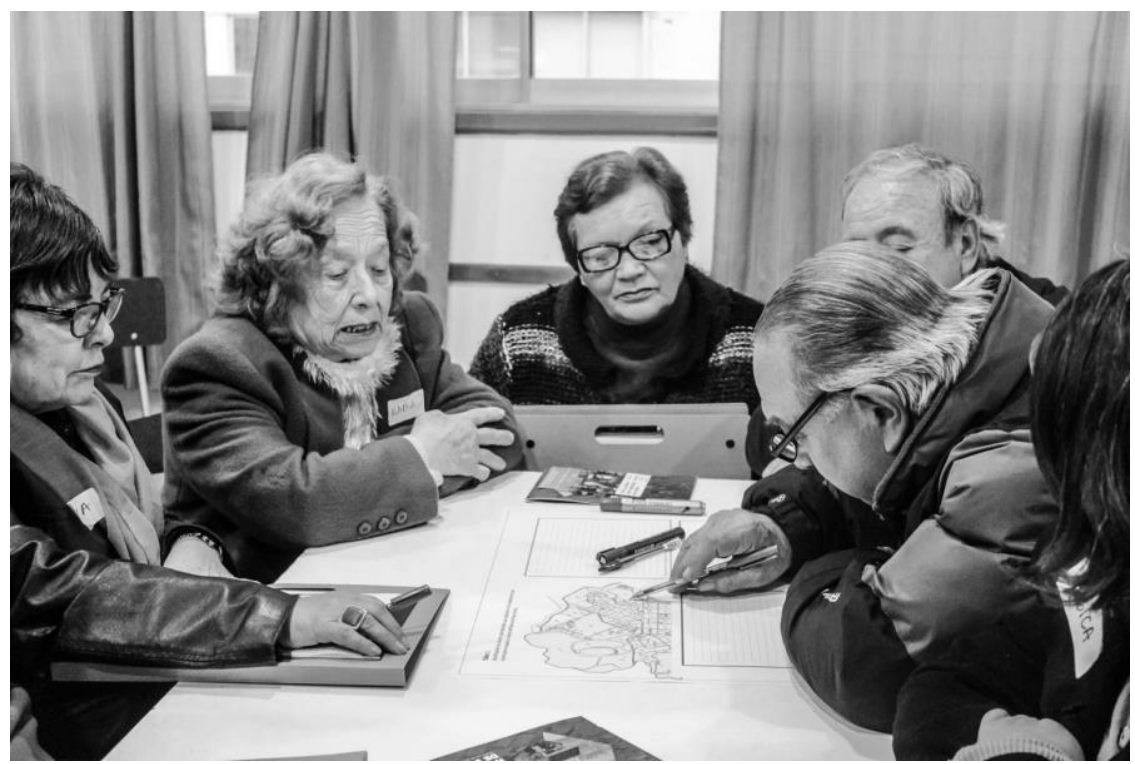

Fuente: Fotografías de María Paz Huenul Colarte - Equipo de Investigación.

Este primer ejercicio, derivó en la construcción de una Cartografía Participativa del Patrimonio Valorado - CPPV. Un registro por categorías que contiene aquellos lugares donde subyacen los valores patrimoniales del barrio, obtenido como expresión de los acuerdos logrados al integrar el trabajo de los grupos de discusión en un solo documento.

Luego, con el propósito de descubrir las relaciones existentes entre los distintos lugares identificados y graficados en la CPPV, se llevó a cabo un ejercicio de puesta en valor del pasado, a objeto de prefigurar, preliminarmente, escenarios futuros y proponer acciones tendientes a generar una toma de conciencia, por toda la ciudadanía, respecto de los lugares valiosos identificados y así, fortalecer su condición heredable; se construyó, en base a la misma técnica de grupos de discusión, una Cartografía Prospectiva del Patrimonio Sistematizado - CPPS.

En una segunda fase, utilizando la información obtenida durante el proceso de sistematización, el grupo de vecinos diseñó tres itinerarios temáticos, según las categorías de valor establecidas, recomendadas por Ballart (2001, pág. 20) -valor asociativo simbólico, valor estético formal y valor de uso social-, a objeto de orientar un ejercicio de observación directa, recorriendo el espacio público, visitando cada uno de los lugares identificados e interpretando la realidad construida y comprendida previamente, acudiendo sólo a la memoria; esta vez, en su estado presente y en una relación dialéctica con el espacio ciudadano. El ejercicio consistió en llevar a 
cabo un diagnóstico in situ respecto de los valores patrimoniales del barrio, expresado en el estado de conservación de las manifestaciones físicas y simbólicas identificadas y que se decidió estuvieran presentes en el recorrido. De esta manera, los vecinos quedaron en condiciones de prefigurar escenarios probables que sirvieron de base a la formulación de acciones prospectivas, en virtud de lo observado.

Figura 2. Excursión por el barrio. La persona observa (mira pensando), registra su experiencia con el entorno a partir de su forma de ver, para posteriormente aportar a la comprensión colectiva.

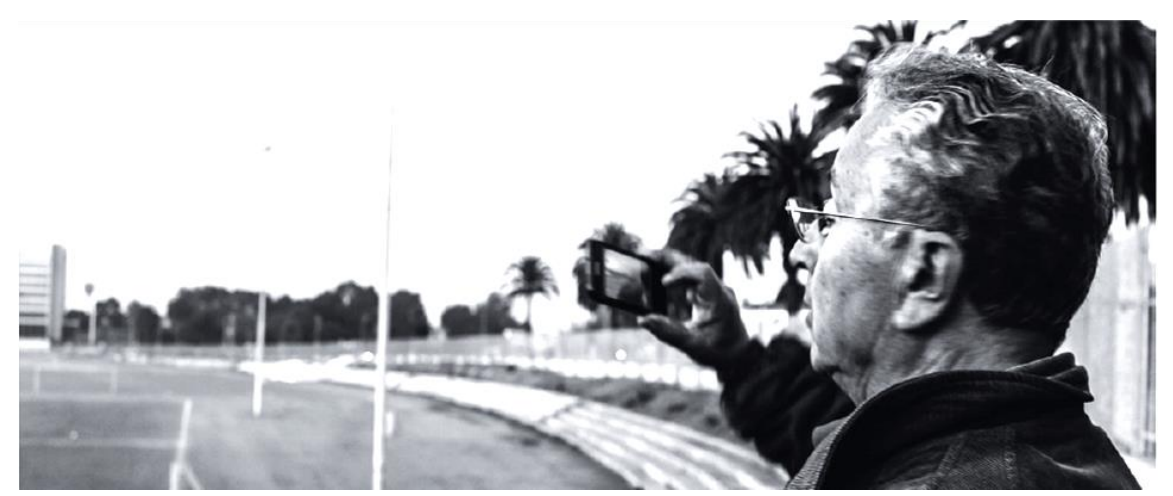

Fuente: Fotografías de María Paz Huenul Colarte - Equipo de Investigación

Como resultado de esta experiencia, se construyó fichas de registro individual del patrimonio y sus valores, las que luego integraron un documento compilatorio con el registro del patrimonio de Playa Ancha Bajo, identificado y valorado por sus habitantes.

Las fichas contienen la identificación de cada uno de los participantes, una identificación de cada lugar seleccionado (cada participante debía escoger dos lugares de todos aquellos visitados), la valoración prospectiva, fotografías inéditas pertenecientes al patrimonio gráfico familiar que permitieran el encuentro con experiencias pasadas y fotografías hechas durante el recorrido, en los mismos lugares escogidos. Se obtuvo un total de cuarenta y cuatro fichas de lugares que los habitantes consideraron valiosos, obtenidas de los veintidós vecinos que completaron el proceso. 
Tabla 2. Fichas de Registro Individual del Patrimonio Valorado.

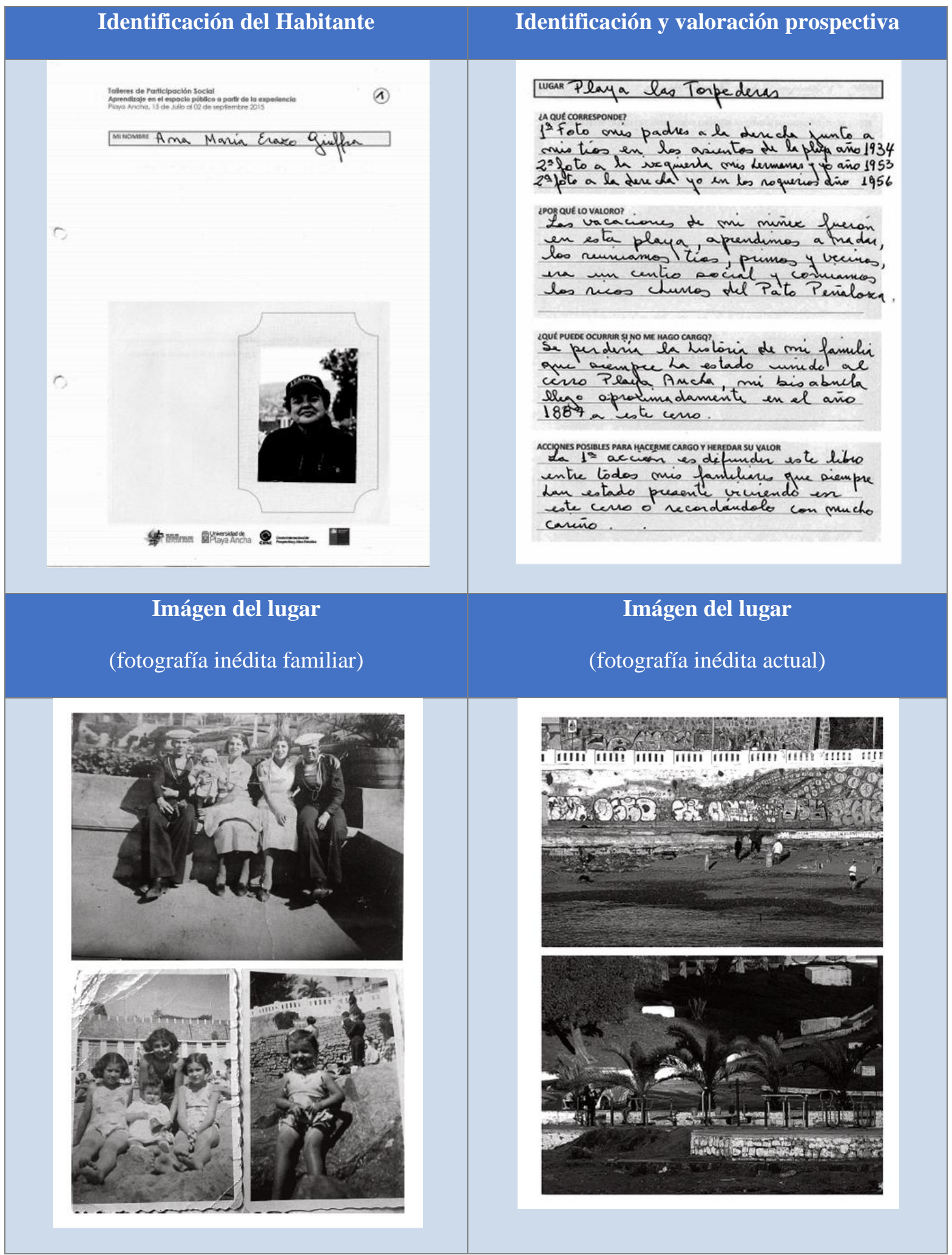

Fuente: Los habitantes del barrio, mediante consentimiento informado, ponen a disposición del investigador principal, fotografías e información obtenida del proceso por cada uno. 
Finalmente, luego de una prefiguración de escenarios probables y en virtud de un ejercicio de valoración desarrollado por cada uno de los grupos de discusión, se obtuvo tres lugares que, a juicio del conjunto social protagonista de la experiencia, merecían ser atendidos y en virtud de ello y de su estado de conservación, propusieron acciones prospectivas para preservar sus valores patrimoniales. Para ello, se trabajó con una prefiguración individual y colectiva, a partir de los escenarios probables establecidos en la fase diagnóstica; prefigurando esta vez, a partir de objetivos comunes, escenarios deseables y posibles logrando propuestas concretas tendientes a actuar sobre el futuro del patrimonio cultural del barrio.

\section{Discusión de resultados}

\section{Sistematización y aprendizaje a partir de la experiencia}

A partir de la definición de ciencia que promueve la investigación-acción participativa (Schmelkes, 2011), entendida como un producto cultural del intelecto humano que responde a necesidades colectivas concretas, que varían conforme a los intereses y objetivos de quienes persiguen la generación de conocimiento y cuya producción se encuentra orientada hacia la preservación y fortalecimiento de la sociedad, se desmitifica la condición científica del conocimiento, cuya generación es atribuible solamente a un segmento facultado para ello.

En este sentido, en la generación de conocimiento, asume un rol fundamental el conjunto social, donde el estudio de la realidad, la identificación de problemas y/o necesidades, no queda alojada fuera de ella. De esta forma, la generación de conocimiento se va gestando como una toma de conciencia colectiva, compartida y participativa sobre la realidad, en base a un proceso profundamente educativo, de momento que "esa conciencia, supone e implica necesariamente una conciencia sobre la necesidad de la transformación. Precisamente porque el conocimiento se genera en el proceso de transformación de la realidad”' (Schmelkes, 2011).

La importancia de este proceso radica en promover una metodología de investigación que, en este estudio, permitió ir más allá de la generación de conocimiento subjetivo, basado solamente en un proceso que va de lo sensitivo a lo perceptual, logrando trascender, superando el voluntarismo, generando condiciones que permitieron resultados objetivos, reales y que impactan efectivamente en el devenir del grupo humano sujeto de investigación, sobre la base de un enfoque de acción transformadora de la realidad.

Esta experiencia hizo posible la construcción de conocimiento compartido, pero además, permitió que los individuos reconocieran su capacidad de transformar la realidad, considerando 
para ello, prácticas que lograron a la postre, motivar y favorecer una conformación orgánica permanente, como parte de un proceso a partir del cual la comunidad de Playa Ancha, hoy, es capaz de organizarse, con el propósito de transitar desde una experiencia de participación educativa a una forma de vida.

La metodología aplicada, ha permitido a los vecinos del barrio transferir su experiencia, más allá del objetivo que se han propuesto, en tanto fortalecer el patrimonio cultural local, logrando instalar en el lugar y en el grupo humano que lo habita, una forma de educar y de educarse de manera permanente. Transcurridos algunos años de la activación del proceso transformacional, se han fortalecido iniciativas como la asociación Salvemos Las Torpederas y el Patrimonio del borde costero de Playa Ancha o palzawaddington.cl y han surgido nuevas organizaciones, como la Plataforma Patrimonial de Playa Ancha, Mujeres en Acción o los Amigos de Plaza Waddington, lideradas por vecinos que han participado del proceso y que, a su vez, han logrado levantar y financiar algunas de las iniciativas surgidas producto de ello y organizar importantes espacios de discusión y construcción de conocimiento como el Primer Congreso Zonal Centro de Barrios Patrimoniales (2018). Congreso que recibió más de 50 ponencias y reunió a más de 200 participantes, incluidas autoridades comunales y regionales y Diputados de la República.

En este sentido, tal como señala Benavides (2004 b), la sistematización, como generadora de conocimiento y orientada, en esta oportunidad, a la formación de valores patrimoniales en un grupo de personas situadas en un lugar específico, aparece como una metodología que se ajusta a la necesidad de responder al objetivo de conocer la realidad, "lugarizarla"(González, 2009) ${ }^{3}$ y humanizarla a partir de un proceso de interpretación crítica de una o varias experiencias. Sin embargo, como resultado de la experiencia se pudo comprobar además, que esta forma de llevar a la práctica la investigación-acción participativa, posee un valor agregado como instrumento gravitante en el proceso y obtener conclusiones teóricas y prácticas al servicio de fases ulteriores centradas en monitorear la experiencia, transferirla y reproducirla.

Luis Benavides (Sistematización. Para aprender a partir de la práctica, 2004 b) define la sistematización como: "un proceso de adquisición de conocimiento que hace posible rescatar, descubrir, ordenar, jerarquizar, interpretar y reflexionar sobre las experiencias, conociéndolas no como experiencias aisladas sino en contextos y en procesos dinámicos y que nos permite modificar,

\footnotetext{
3 "Llugarización”, concepto acuñado por Francisco González Cruz, "como la nueva naturaleza de los lugares, en el marco de una visión menos materialista y economicista de la realidad, es decir más humana e integral, puede abrir nuevas esperanzas para comprender el tema del desarrollo humano sustentable desde una perspectiva más integral y comprensiva, más holística".
} 
mejorar o adecuar prácticas entre todos los agentes y actores sociales involucrados en ellas " (pág. 2).

Aplicada la sistematización o método de aprendizaje a partir de la práctica, los habitantes de Playa Ancha lograron descubrir y redescubrir la experiencia de habitar, en una relación pasado, presente, futuro y mediante la observación -con todos los sentidos atentos y haciendo de las acciones de cada día, experiencias de aprendizaje en el espacio público- lograron interpretar la realidad, descomponiendo la forma en que se construye, para luego, haciendo acopio del conocimiento construido previamente y utilizando lo aprendido para proponer mejoras, iniciaron un proceso transformacional que en una segunda fase del estudio pudo ser monitoreado y reforzado, centrando la intervención en estrategias pedagógicas orientadas a la formulación y ejecución de proyectos.

Fue así que el barrio de Playa Ancha Bajo, en tanto realidad cargada de valores heredables, pudo ser re-conocido dentro de un sistema dinámico de relaciones entre distintas realidades que confluyeron y generaron diversos contextos; los que, puestos en relación e interacción permanente, han ido conformando formas de vida que se expresan en manifestaciones culturales -físicas y simbólicas- y que a su vez, han ido construyendo un entorno a partir de huellas que finalmente son capaces de orientar el devenir de quienes participan y experimentan día a día el proceso de construcción de dicha realidad.

\section{La dialéctica en la comprensión del espacio}

Si bien la dialéctica hasta el siglo dieciocho, aparece más bien vinculada a la lógica y a la epistemología bajo la forma de debate o discusión de ideas, a partir de un diálogo que persigue relacionarlas entre sí, con el propósito de desarrollar una comprensión sobre la realidad; algunos autores señalan que no existe la posibilidad de un encuentro auténtico de miradas, de momento que la dialéctica opera sobre una base ideológica que le imprime un carácter instruccional, donde se pierde la posibilidad de construir interpretaciones holísticas y la comprensión de la realidad se realiza a partir de contextos aislados, que no permiten vincular ideas, conceptos, miradas y objetivos comunes (Abellán, 2012).

Pero, como resultado de la experiencia, se pudo comprobar que en la comprensión del espacio, a partir de la vivencia que el individuo experimenta al observarlo, en directa relación con la manifestación física y simbólica que le da cuerpo (lugar) -aun cuando en la práctica se encuentre acompañado y con la posibilidad de dialogar con otros- primero es individuo en relación con el 
espacio y luego es parte de la discusión respecto de su significado. Con todos sus sentidos atentos, a partir de su experiencia, observa, recibe los estímulos del medio y desarrolla un proceso mental que lo lleva a percibir con éxito, desde su individualidad, aquellas relaciones que estructuran una comprensión de sus vivencias. Aunque esta interpretación es parcial y luego puede y debe ser compartida con el fin de llevar a cabo comprensiones colectivas, en un primer momento, el individuo fue capaz de concentrarse en su individualidad, observando y viviendo lo que ocurría a su alrededor, para finalmente aportar a la construcción de un conocimiento, una significación y una valoración compartida.

En este ejercicio, los vecinos fueron capaces de reconocer elementos que les permitieron descomponer la realidad y abstraerla, jerarquizando y sintetizando los elementos que la definen, proporcionando valor a ciertos elementos que configuraron su propia comprensión de ella. De esta forma los registros elaborados por cada participante, permitieron comprobar que cada cual percibe la realidad de una forma distinta y, en virtud de su propia experiencia, le asigna valor a cada lugar reconocido. Sin embargo, la dimensión cognoscitiva que le permitió comprenderlo y significarlo con mayor profundidad, surge de la experiencia de construcción de conocimiento compartido de la cual tuvo la oportunidad de formar parte.

\section{La dialógica en la construcción de conocimiento compartido}

De regreso en el grupo, de regreso en el espacio de reflexión colectiva y participativa centrada en el diálogo, luego de la experiencia vivida desde su propia realidad, atentos ahora a la realidad colectiva, los vecinos se avocaron a contribuir con su registro, con su reflexión, con su interpretación y con su comprensión; a un registro, una reflexión, una interpretación y una comprensión común.

De esta manera, se puso en valor, esta vez, la dialógica de las relaciones sociales, a partir de la dialéctica del espacio, pero como un método de construcción compartida de conocimiento, favoreciendo una "búsqueda comunitaria (dialogada) y que se alcanza, confirma y realiza fundamentalmente en comunidad”.(Abellán, 2012, pág. 111)

De la atención en el objeto aislado para la comprensión de los mensajes percibidos en un deambular por el espacio público (dialéctica), se pasó a un ejercicio que permitió vincular ideas y conceptos para asistir esta vez, a un acto colectivo de construcción de conocimiento compartido, donde primó el diálogo entre sujeto y realidad (dialógica), donde cada uno de los interlocutores aportó con lo suyo y desde lo suyo. Superada la experiencia basada en el silencio de la relación 
sujeto-objeto, se hizo presente la dinámica de hablar y escuchar, de compartir y construir en comunidad.

En cuanto al desempeño de los individuos, es importante señalar, a la luz del fenómeno estudiado, que los seres humanos transcurren en una dinámica que necesariamente les sugiere pasar de lo individual a lo colectivo y luego de lo colectivo a lo individual; por cuanto, en la lógica de los aprendizajes que promueve la educación permanente, de los estados de observación y toma de conciencia individual, el individuo debe necesariamente pasar a un estado de socialización de su mirada, su reflexión y su comprensión de la realidad y, de esta manera, prefigurar el futuro para actuar sobre él. Siempre en directa relación con su comunidad. En este sentido, todo ejercicio de valoración colectiva, se vuelve dependiente de la capacidad que cada individuo desarrolle de interpretar la realidad y tomar conciencia del valor que tiene para él. Siendo de máxima relevancia que en esta dinámica se desarrolle una capacidad que permita la 'autodidaxia'. (Faure, 1972)

En el informe liderado por Edgar Faure (1972), la UNESCO, recomendando la Educación Permanente como principio para la elaboración de políticas educativas, señala que para ello, se debe propender a diversificar las formas de educar, permitiendo y promoviendo el educarse por sí mismo, difundiendo la experiencia de aprendizaje como una práctica centrada en el principio de la autodidaxia.

Está comprobado que la autodidaxia no nace en el individuo en forma espontánea y que se requiere procesos tendientes a facilitar el aprendizaje, sobre la base de un enfoque pedagógico que los facilitadores o promotores educativos deben dominar, si quieren transmitirlo a la comunidad como el ideal que suscita. Para lograr la capacidad de educarse por sí mismo, el individuo debe adoptar una actitud frente al desarrollo humano como es la de hacer de la educación permanente una forma de vida.

A este enfoque pedagógico se le denomina aprender a aprender, lo que implica una adquisición de hábitos de trabajo y un despertar de la conciencia, detonando motivaciones que es necesario provocar, no tan solo en estudiantes que asisten a la educación formal, si no desde ellos, en la comunidad toda para favorecer un desarrollo humano más próspero.

En este contexto, si bien los resultados de este estudio fueron positivos, en tanto se logró fortalecer la identidad cívica en un sector de la ciudad de Valparaíso, instalando ciertos liderazgos en actores que desde hoy se configuran como potenciales promotores educativos, es dable reforzar lo observado en la formulación de esta investigación respecto de la necesidad de promover y apoyar 
este tipo de acciones educativas, a partir de una política con enfoque social. Política que contemple la formación de promotores educativos, líderes sociales capaces de instalar la filosofía de la educación permanente como parte del sistema social. "Para poder concretar las aspiraciones de la autodidaxia, cada uno debe poder encontrar no sólo en la escuela y en la Universidad, sino en todos los lugares y circunstancias donde sea posible, modalidades e instrumentos adecuados para hacer del estudio personal una actividad fecunda. La adquisición de los mecanismos de aprendizaje autónomo y la disponibilidad de amplios medios auxiliares aumentan mucho la eficacia del estudio solitario. A este respecto, si no se quiere permanecer al nivel de meras declaraciones de intención, interesa que en los presupuestos de educación se asignen créditos suficientes para desarrollar la autodidaxia”. (Faure, 1972, pág. 270)

En este sentido, cobra importancia un aspecto fundamental de la dialógica en su dimensión antropológica y es que pone en valor el brotar de la conciencia, que en un acto reflejo, le permite darse cuenta, de quién es y de lo que es. Lo que va aparejado del descubrimiento del yo y la importancia de reconocer la existencia de un otro. En este despertar de la conciencia, radica precisamente el valor que tiene en el individuo la capacidad de relacionarse con el mundo que lo rodea y construir desde él y junto con él, un nosotros que finalmente otorga valor y sentido a su existencia.

\section{Conclusiones}

Aplicada la metodología propuesta en la investigación objeto de este artículo, quedó comprobado que la formación de valores patrimoniales, basada en un enfoque sustentado en la acción-participativa y aplicada en el lugar donde éstos se manifiestan, permite lograr su reconocimiento como algo propio y digno de ser conservado. Por supuesto, en la medida que aquella percepción se oriente mediante un proceso educativo centrado en aportar al conocimiento que haga posible una justa interpretación de dichos valores y la formación de una actitud prospectiva que permita actuar anticipada y responsablemente sobre ellos.

La educación permanente, fundada en la participación social y la prospectiva, permite consolidar procesos donde los grupos sociales significan la condición patrimonial de los bienes culturales que forman parte de su entorno; los que, a partir de la acción de construir y reconstruir 
un conocimiento compartido y de procesos ligados a la sistematización como metodología de aprendizaje a través de la práctica, comienzan a conocer y re-conocer como parte de su identidad.

Con todo, se comprueba que el conocimiento de los bienes culturales y el re-conocimiento de su valor patrimonial, proporcionan las bases necesarias para su entendimiento y para orientar una adecuada gestión de conservación, pero se concluye además, que la valoración del patrimonio cultural permite fortalecer una identidad social y cultural cargada de significado, capaz de generar interés y motivación en la comunidad por fortalecerlo y transferirlo a las nuevas generaciones.

Se concluye que la educación, en este sentido, debe permitir a las personas desarrollar sus capacidades individuales y desde ahí satisfacer las necesidades y demandas de la sociedad, en términos de proporcionar a los individuos la capacidad y posibilidad de actuar en comunidad en favor del fortalecimiento de su identidad cívica.

En este sentido, los avances que el Estado pueda lograr en cuanto a conservación de su Patrimonio Cultural y la preservación y fortalecimiento del mismo, se vuelven dependientes de la capacidad que éste tenga, de promover una educación entendida como un proceso permanente, abierto y participativo, que supere los límites de la educación formal, permitiendo el acceso de todo el conjunto social a proyectos de desarrollo que favorezcan la concienciación de sus valores.

La educación debe necesariamente promover la construcción de conocimiento compartido en torno a aquellos tangibles e intangibles que son expresión de la cultura nacional e internacional, pero sin perder de vista la cultura local como expresión de valor que surge de las comunidades, que subyace en el entorno y que sólo es posible valorar, en la medida que se fortalezca el vínculo de los habitantes con su territorio. Comenzando por fomentar una experiencia educativa con aquellos lugares donde se desarrolla cada una de las expresiones culturales que proporcionan identidad a un determinado grupo humano, para luego, recién desde ahí, hacer una comprensión y una valoración de las expresiones culturales que identifican a la Nación en un contexto global.

Esta investigación refuerza la importancia que reviste la necesidad de extender la función formativa y socializadora de la educación a otros espacios como la ciudad. Los beneficiarios no pueden ser sólo niños y adolescentes, la educación debe estar orientada a promover procesos de educación que favorezcan a toda la sociedad, debe ser entendida como un proceso continuo, que abarque además, todos los espacios donde subyace una carga simbólica capaz de favorecer aprendizajes culturalmente significativos y que en tal sentido, se oriente a promover aprendizajes 
centrados no tan solo en el conocer, sino además en el saber ser, saber hacer y fundamentalmente, saber convivir.

De la investigación realizada se desprende además, que esta forma de ver la educación va de la mano con la conservación del patrimonio, entendida como un proceso que persigue transferir, de generación en generación, un legado de tradiciones que dan vida al desarrollo cultural del conjunto social que lo valora. De ahí que la educación permanente se vuelve pieza clave en la transmisión de estos valores, ya que es ella la que permite rescatarlos y hacer que perduren en el tiempo instalándolos en el grupo humano que los representa, formando en ellos una actitud prospectiva que les permite hacerse cargo de su transferencia.

Si se logra producir una sinergia entre la filosofía de la educación permanente, la promoción del conocimiento y la formación de valores patrimoniales, como herramientas fundamentales para la conservación de las expresiones culturales donde subyace la identidad cultural de las comunidades, será posible, no tan sólo asumir con eficacia la responsabilidad respecto de la protección e incremento del Patrimonio Cultural de la Nación, sino además será posible convertirlo en un elemento clave para la formación integral de las personas, para su desarrollo emocional y propiciar a través de ello, una fructífera interacción y cohesión social frente a la tarea de identificar, registrar y transferir los valores de una determinada cultura, generación tras generación.

En cuanto a una política de desarrollo del patrimonio, bajo la óptica de la educación permanente, el compromiso de los habitantes y la participación social aparecen también como piezas clave, a la hora de elaborar planes de manejo de los llamados Centros Históricos, toda vez que la conservación de las ciudades y barrios históricos, concierne en primer lugar a sus habitantes.

De esta forma se llega a la importancia que adquiere la relación educación (permanente) y espacio (lugar), en términos de desarrollar procesos de aprendizaje cognitivos y axiológicos, en favor de la significación y la preservación del patrimonio cultural, así como de la transferencia de sus valores y su adopción por toda la ciudadanía.

En tal sentido, esta investigación se hizo cargo y promueve una forma de aprendizaje a través de la formación de valores patrimoniales llevando a cabo una acción transformadora, en el contexto de las relaciones existentes entre los habitantes de un territorio culturalmente significativo y de ellos con el lugar o espacio cultural donde subyacen dichos valores.

Esta experiencia de habitar, estudiar y comprender el lugar como una expresión cultural que da cuenta de la relación indivisible entre el espacio y el ser humano que lo habita, permitió 
comprobar que para preservar y garantizar la transferencia de los valores identitarios de una determinada cultura a las nuevas generaciones, se hace necesario impulsar acciones educativas vinculadas social y espacialmente con el entorno, bajo una nueva concepción de la educación que se construye a partir de procesos que involucran el sistema social en forma integral, a partir de un necesario vínculo con el lugar donde se desarrolla una determinada cultura y donde su puesta en valor radica precisamente, en su condición de espacio social, culturalmente habitado, construido y vivido por un determinado grupo humano que persigue crecer y desarrollarse a partir de sus diferencias y de lo que le es propio.

Los grupos humanos sienten, interpretan, reconocen, construyen y viven en un determinado lugar, en directa relación con sus características físicas, simbólicas y sociales, las que hacen síntesis en una dimensión humana del espacio construido que, en sí, construye su propia existencia y le proporciona significado y sentido a al devenir de la humanidad.

Se logró comprobar la importancia que trae consigo la capacidad que deben lograr los grupos humanos de desarrollar su sentido de pertenencia e identificarse con propósitos comunes, creando y fortaleciendo lazos entre sí y con el lugar donde desarrollan su vida; a fin de alcanzar los acuerdos necesarios para que en una toma de conciencia colectiva, se pueda llegar a un consenso de lo que el lugar es y lo que podría llegar a ser para un nosotros. Sólo así, será posible tomar decisiones respecto de aquellos valores que se desee conservar y aquellos que se desee transformar a favor de ese futuro que se pretende construir.

Como producto relevante, se obtuvo un insumo para el diseño de estrategias orientadas a la transformación de la realidad, a objeto de activar procesos de participación ciudadana que posibiliten la creación de consenso en torno a las prioridades educativas de aquellas comunidades que tienen el privilegio de habitar lugares cargados de valor y emprender estrategias que les permitan a su vez, transformar esos lugares en sujeto y objeto de aprendizaje.

Sin embargo, se configura como conclusión importante de este estudio que para lograr aún mejores resultados, los habitantes deben tener la posibilidad de aprender a leer la ciudad, desarrollar la capacidad de observar tras la dinámica continua, superando aquellas barreras que impiden poner atención más allá del lugar donde viven y penetrar aquellas manifestaciones físicas y simbólicas que se encuentran en el entorno. Los habitantes deben alcanzar niveles de compromiso, que les permitan poner en práctica una lectura crítica del territorio, a partir del uso efectivo de los espacios que éste ofrece y participar en su construcción, influyendo directamente en su devenir, a partir de 
acciones concretas que se encuentren al alcance de cada uno, individual y colectivamente. De esta forma, invocar y fortalecer la identidad y el sentido de pertenencia responsable que puedan lograr procesos como el que se llevó a cabo mediante este estudio.

La participación activa de los habitantes en la conservación y fortalecimiento del patrimonio de una Nación es relevante. El habitante es el interlocutor válido, es la voz autorizada que carga con la experiencia necesaria para manifestarse respecto de los valores que subyacen en el espacio físico y simbólico en el que habita; es el responsable de conocer los significados, resignificar, pensar y repensar en comunidad cada lugar. Es quien debe apropiarse de lo que le es suyo, de lo que tiene, de aquello con lo que cuenta y desde ahí, en la suma de voluntades, apropiarse de su futuro, formulando proyectos que le permitan trabajar, desde hoy, en un mejor futuro para su barrio y, desde allí, en un mejor futuro para la ciudad que lo educa y que a su vez, se educa, a partir de su crecimiento individual y social como ser humano.

\section{Referencias Bibliográficas}

Abellán, A. (2012). La Dialéctica y la Dialógica. Revista del Humanismo Español e Iberoamericano. Mar Oceana(31), 97-125.

Ballart, J., \& i Tresseras, J. (2001). Gestión del Patrimonio Cultural. Barcelona: Ariel Patrimonio.

Benavides, L. (2002). Espacios y conceptos educativos. Taller Internacional sobre infraestructura educativa (págs. 1-2). Guadalajara: s.e.

Benavides, L. (2004 a). Metodología Prospectiva. Apuntes: Doctorado en Educación Permanente, Centro Internacional de prospectiva y Altos Estudios. Puebla, México: CIPAE.

Benavides, L. (2004 b). Sistematización. Para aprender a partir de la práctica. Apuntes: Doctorado en Educación Permanente, Centro Internacional de prospectiva y Altos Estudios. Puebla, México: CIPAE.

CIDPA. (2005). Propuesta de intervención del currículum escolar de alumnos de establecimientos municipalizados de la comuna de Valparaíso. Valparaíso: I. Municipalidad de Valparaíso.

CIPAE. (2012). Qué es la Educación Permanente. Recuperado el 2014, de http://www.cipae.edu.mx: http://www.cipae.edu.mx/index.php/educacion-permanente

Ciudades Educadoras, A. I. (1990). Carta de Ciudades Educadoras. Recuperado el 2020, de https://www.edcities.org: https://www.edcities.org/wp-content/uploads/2020/11/ES_Carta.pdf

Dámaris, N. (2014). Valparaíso ¿Ciudad educadora?. Análisis sobre el uso del patrimonio con fin educativo. Recuperado el 2015, de https://prezi.com: https://prezi.com/66-_sqo4kcgd/valparaisociudad-educadora-analisis-sobre-el-uso-del-pa/ 
Devia, M. D. (2003). Educación Patrimonial o Patrimonio para la Educación. Sociedad: Re-visión de Escenarios(3), Universidad de Playa Ancha, 49-57. Valparaíso, Chile: Universidad de PLaya Ancha.

Faure, E. (1972). Aprender a Ser, La educación del futuro. Paris, Francia: Alianza UNESCO. Recuperado el 2015, de http://unesdoc.unesco.org/images/0013/001329/132984s.pdf

González, F. (2009). Desarrollo Humano Sustentable. Polis. Revista de la Universidad Bolivariana, 8(2), 57.

Heiddeger, M. (2004). Construir, Habitar, Pensar. Recuperado el 2015, de http://www.geoacademia.cl: http://www.geoacademia.cl/docente/mats/construir-habitar-pensar.pdf

Mondaca, R. (1997). La ciudad educadora. Un concepto y una propuesta con historia. Medellín: Corporación Región.

Petrus, A. (1997). Pedagogía Social (comp.). Concepto de Educación Social. Barcelona: Ariel.

Ruiz Botero, L. (2001). La sistematización de prácticas. Recuperado el 2014, de www.oei.es: www.oei.es/historico/equidad/liceo.PDF

Schmelkes, S. (2011). Fundamentos teóricos de la Investigación Participativa, Biblioteca CREFAL. Recuperado el 2015, de http://www.virtual.unal.edu.co:

http://www.virtual.unal.edu.co/cursos/humanas/mtria_edu/2021085/und_2/pdf/fundamentos.pdf

Suazo, M. (2003). Las funciones sociales de la Educación. Sociedad: Re-visión de Escenarios(3), Universidad de Playa Ancha, 16-24. Valparaíso, Chile: Universidad de Playa Ancha.

UNESCO. (1972). Convención para la proteccción del Patrimonio Mundial. Recuperado el 2020, de https://whc.unesco.org: https://whc.unesco.org/archive/convention-es.pdf

UNESCO. (2014-2019). Casco Histórico de la Ciudad Portuaria de Valparaíso. Recuperado el 2020, de https://whc.unesco.org: https://whc.unesco.org/en/list/959/documents/

Valles, M. (2003). Técnicas Cualitativas de la Investigación Social - Reflexión metodológica y práctica profesional. Madrid: Síntesis S.A.

Revista RedCA, Vol. 4, Núm. 10 (junio-septiembre 2021) Universidad Autónoma del Estado de México, ISSN-e: 2594-2824 